\title{
Effective irrationality measures for quotients of logarithms of rational numbers
}

\author{
Yann Bugeaud
}

\begin{abstract}
We establish uniform irrationality measures for the quotients of the logarithms of two rational numbers which are very close to 1. Our proof is based on a refinement in the theory of linear forms in logarithms which goes back to a paper of Shorey.

Keywords. Linear forms in logarithms, Baker's theory, irrationality measure.
\end{abstract}

2010 Mathematics Subject Classification. 11J82, 11J86

\section{Introduction and result}

Let $\xi$ be an irrational real number. The real number $\mu$ is an irrationality measure of $\xi$ if, for every positive $\varepsilon$, there are a positive number $C(\xi, \varepsilon)$ and at most finitely many rational numbers $p / q$ with $q \geq 1$ and

$$
\left|\xi-\frac{p}{q}\right|<\frac{C(\xi, \varepsilon)}{q^{\mu+\varepsilon}} .
$$

If, moreover, the constant $C(\xi, \varepsilon)$ is effectively computable for every positive $\varepsilon$, then $\mu$ is an effective irrationality measure of $\xi$. We denote by $\mu(\xi)$ (resp. $\mu_{\text {eff }}(\xi)$ ) the infimum of the irrationality measures (resp. effective irrationality measures) of $\xi$. It follows from the theory of continued fractions that $\mu(\xi) \geq 2$ for every irrational real number $\xi$ and an easy covering argument shows that there is equality for almost all $\xi$, with respect to the Lebesgue measure.

The following statement is a straightforward consequence of Baker's theory of linear forms in logarithms (see e.g. [Wa00] and the references therein). By definition, two positive rational numbers are multiplicatively independent if the quotient of their logarithms is irrational.

Theorem 1.1 Let $a_{1}, a_{2}, b_{1}, b_{2}$ be positive integers with $a_{1}>a_{2}$ and $b_{1}>b_{2}$. Assume that $a_{1} / a_{2}$ and $b_{1} / b_{2}$ are multiplicatively independent. There exists an absolute, effectively computable, constant $C$ such that

$$
\mu_{\mathrm{eff}}\left(\frac{\log \left(a_{1} / a_{2}\right)}{\log \left(b_{1} / b_{2}\right)}\right) \leq C\left(\log a_{1}\right)\left(\log b_{1}\right) .
$$

The purpose of this note is to show how a known refinement in the theory of linear forms in logarithms in the special case where the rational numbers are very close to 1 , which goes back to Shorey's paper [Sho74], allows one to considerably improve Theorem 1.1 in this special case. Several spectacular applications to Diophantine problems and to Diophantine equations of this idea of Shorey have already been found; see for example [Wa78, Wa00] and the survey [Bu08]. Quite surprisingly, it seems that it has not yet been noticed that it can be used to give uniform upper bounds for irrationality measures of roots of rational numbers (see [Bu16]) and of quotients of logarithms of rational numbers, under some suitable assumptions. 
Our main result is the following.

Theorem 1.2 Let $a_{1}, a_{2}, b_{1}, b_{2}$ be positive integers such that

$$
\max \left\{16, a_{2}\right\}<a_{1}<6 a_{2} / 5 \text { and } \max \left\{16, b_{2}\right\}<b_{1}<6 b_{2} / 5 \text {. }
$$

Define $\eta$ by $a_{1}-a_{2}=a_{1}^{1-\eta}$ and $\nu$ by $b_{1}-b_{2}=b_{1}^{1-\nu}$. If $a_{1} / b_{1}$ and $a_{2} / b_{2}$ are multiplicatively independent, then we have

$$
\mu_{\mathrm{eff}}\left(\frac{\log \left(a_{1} / a_{2}\right)}{\log \left(b_{1} / b_{2}\right)}\right) \leq 1+27638 \frac{\left(\log a_{1}\right)\left(\log b_{1}\right)}{\min \left\{\eta \log a_{1}, \nu \log b_{1}\right\}^{2}} .
$$

We display an immediate corollary of Theorem 1.2 which deals with the case $\eta>1 / 2$ and $\nu>1 / 2$. It illustrates the strength of the theory of linear forms in logarithms.

Corollary 1.3 Let $a_{1}, a_{2}, b_{1}, b_{2}$ be positive integers such that

$$
36 \leq a_{2}<a_{1}<a_{2}+\sqrt{a_{1}}, \quad 36 \leq b_{2}<b_{1}<b_{2}+\sqrt{b_{1}}, \quad \text { and } \quad \sqrt{b_{1}}<a_{1}<b_{1}^{2} .
$$

If $a_{1} / b_{1}$ and $a_{2} / b_{2}$ are multiplicatively independent, then we have

$$
\mu_{\mathrm{eff}}\left(\frac{\log \left(a_{1} / a_{2}\right)}{\log \left(b_{1} / b_{2}\right)}\right) \leq 221105
$$

It is apparent from the proof of Theorem 1.2 that the numerical constants in (1.2) and (1.3) can be reduced (roughly, divided by 3 ) if $a_{1}$ and $b_{1}$ are sufficiently large. No particular significance has to be attached to the numerical constant $6 / 5$ in (1.1).

Let $a, b$ and $d$ be positive integers with $a \neq b$ and $\max \{a, b\}<d$. Under certain conditions, Rhin [Rh83] (see also [RhTo86]) obtained explicit upper bounds for

$$
\mu_{\mathrm{eff}}\left(\frac{\log (1+a / d)}{\log (1+b / d)}\right) .
$$

His approach, which gives better numerical results than ours, heavily uses the fact that the two rational numbers $a / d$ and $b / d$ have the same denominator. It seems to us that Theorem 1.2 , which applies without any specific restriction on the denominators $a_{2}$ and $b_{2}$ of the rational numbers, is new and cannot be straightforwardly derived from the methods of [Rh83, RhTo86].

\section{Proof of Theorem 1.2}

We reproduce with some simplification Corollary 2.4 of Gouillon [Gou06] in the special case where the algebraic numbers involved are rational numbers. We replace his assumption $E \geq 2$ by $E \geq 15$, to avoid trouble with the quantity $\log \log \log E$ occurring in the definition of $E^{*}$ in Corollary 2.4 of [Gou06], which is not defined if $E$ is too small.

Theorem G Let $a_{1}, a_{2}, b_{1}, b_{2}$ be positive integers such that $a_{1} / a_{2}$ and $b_{1} / b_{2}$ are multiplicatively independent and greater than 1 . Let $A$ and $B$ be real numbers such that

$$
A \geq \max \left\{a_{1}, \mathrm{e}\right\}, \quad B \geq \max \left\{b_{1}, \mathrm{e}\right\} .
$$

Let $x$ and $y$ be positive integers and set

$$
X^{\prime}=\frac{x}{\log A}+\frac{y}{\log B} .
$$


Set

$$
E=1+\min \left\{\frac{\log A}{\log \left(a_{1} / a_{2}\right)}, \frac{\log B}{\log \left(b_{1} / b_{2}\right)}\right\}
$$

and

$$
\log X=\max \left\{\log X^{\prime}+\log E, 265 \log E, 600+150 \log E\right\} .
$$

Assume furthermore that $15 \leq E \leq \min \left\{A^{3 / 2}, B^{3 / 2}\right\}$. Then,

$$
\log \left|y \log \left(a_{1} / a_{2}\right)-x \log \left(b_{1} / b_{2}\right)\right| \geq-8550(\log A)(\log B)(\log X)(4+\log E)(\log E)^{-3} .
$$

It is crucial for our proof that the dependence on $X$ comes through the factor $(\log X)$ and not through $(\log X)^{2}$, as in [LMN95]. Instead of Theorem G we could use an earlier result of Waldschmidt [Wa93] (see also Theorem 9.1 of [Wa00]), but the numerical constants in (1.2) and (1.3) would then be slightly larger.

Proof of Theorem 1.2. Our aim is to estimate from below the quantity

$$
\left|\frac{\log \left(a_{1} / a_{2}\right)}{\log \left(b_{1} / b_{2}\right)}-\frac{x}{y}\right|
$$

for large positive integers $x, y$. We will establish a lower bound of the form

$$
\log \left|y \log \left(a_{1} / a_{2}\right)-x \log \left(b_{1} / b_{2}\right)\right| \geq-C_{1}\left(C_{2}+\log \max \{x, y\}\right)
$$

for some quantities $C_{1}, C_{2}$ which depend at most on $a_{1}, a_{2}, b_{1}$ and $b_{2}$. This will show that $1+C_{1}$ is an irrationality measure for $\log \left(a_{1} / a_{2}\right) / \log \left(b_{1} / b_{2}\right)$.

We apply Theorem $\mathrm{G}$ and set $A=a_{1}$ and $B=b_{1}$.

Observe that

$$
\log \left(a_{1} / a_{2}\right)=\log \left(1+\left(\left(a_{1}-a_{2}\right) / a_{2}\right)\right) \leq\left(a_{1}-a_{2}\right) / a_{2}=a_{1}^{1-\eta} a_{2}^{-1}
$$

thus

$$
\frac{\log a_{1}}{\log \left(a_{1} / a_{2}\right)} \geq\left(\log a_{1}\right) \frac{a_{2}}{a_{1}} a_{1}^{\eta} \geq \max \left\{14, a_{1}^{\eta}\right\},
$$

since $a_{1} \geq 17$ and $a_{1}^{\eta}=a_{1} /\left(a_{1}-a_{2}\right) \geq 6$. Likewise, we check that

$$
\frac{\log b_{1}}{\log \left(b_{1} / b_{2}\right)} \geq \max \left\{14, b_{1}^{\nu}\right\}
$$

Furthermore, since $a_{2} \geq 14$ and $a_{1} \geq 17$, we get

$$
1+\frac{\log a_{1}}{\log \left(a_{1} / a_{2}\right)} \leq 1+\frac{\log a_{1}}{\log \left(1+1 / a_{2}\right)} \leq 1+1.1 a_{2} \log a_{1} \leq a_{1}^{3 / 2}
$$

and a similar upper bound holds for $1+\left(\log b_{1}\right) /\left(\log \left(b_{1} / b_{2}\right)\right)$, thus we have proved that

$$
E:=1+\min \left\{\frac{\log a_{1}}{\log \left(a_{1} / a_{2}\right)}, \frac{\log b_{1}}{\log \left(b_{1} / b_{2}\right)}\right\}
$$

satisfies

$$
15 \leq E \leq \min \left\{A^{3 / 2}, B^{3 / 2}\right\}
$$

Note also that the quantity

$$
E^{\prime}=\min \left\{a_{1}^{\eta}, b_{1}^{\nu}\right\}
$$


satisfies $6 \leq E^{\prime} \leq E$, thus $\left(4+\log E^{\prime}\right) /\left(\log E^{\prime}\right) \leq(4+\log 6) /(\log 6)$. It then follows from Theorem G that

$$
\log \left|y \log \left(a_{1} / a_{2}\right)-x \log \left(b_{1} / b_{2}\right)\right| \geq-27638(\log A)(\log B)(\log \max \{x, y\}+\log E)\left(\log E^{\prime}\right)^{-2},
$$

when $\max \{x, y\}$ is sufficiently large. This lower bound is of the form (2.1), with explicit values for $C_{1}$ and $C_{2}$, thus we have proved that

$$
\mu_{\mathrm{eff}}\left(\frac{\log \left(a_{1} / a_{2}\right)}{\log \left(b_{1} / b_{2}\right)}\right) \leq 1+27638 \frac{\left(\log a_{1}\right)\left(\log b_{1}\right)}{\min \left\{\eta \log a_{1}, \nu \log b_{1}\right\}^{2}} .
$$

This completes the proof of Theorem 1.2.

\section{References}

[Bu08] Y. Bugeaud, Linear forms in the logarithms of algebraic numbers close to 1 and applications to Diophantine equations, Proceedings of the Number Theory conference DION 2005, Mumbai, pp. 59-76, Narosa Publ. House, 2008.

[Bu16] Y. Bugeaud, Effective irrationality measures for roots of rational numbers close to 1 . In preparation.

[Gou06] N. Gouillon, Explicit lower bounds for linear forms in two logarithms, J. Théor. Nombres Bordeaux 18 (2006), $125-146$.

[LMN95] M. Laurent, M. Mignotte et Y. Nesterenko, Formes linéaires en deux logarithmes et déterminants d'interpolation, J. Number Theory 55 (1995), 285-321.

[Rh83] G. Rhin, Sur l'approximation diophantienne simultanée de deux logarithmes de nombres rationnels. In: Approximations diophantiennes et nombres transcendants, Progress in Mathematics, Colloque de Luminy 1982 (4th ed.), Vol. 31, Birkhäuser, Basel (1983), pp. 247-258.

[RhTo86] G. Rhin and Ph. Toffin, Approximants de Padé simultanés de logarithmes, J. Number Theory 24 (1986), $284-297$.

[Sho74] T. N. Shorey, Linear forms in the logarithms of algebraic numbers with small coefficients I, J. Indian Math. Soc. (N. S.) 38 (1974), 271-284.

[Wa78] M. Waldschmidt, Transcendence measures for exponentials and logarithms, J. Austral. Math. Soc. Ser. A 25 (1978), $445-465$.

[Wa93] M. Waldschmidt, Minorations de combinaisons linéaires de logarithmes de nombres algébriques, Canadian J. Math. 45 (1993), 176-224.

[Wa00] M. Waldschmidt, Diophantine Approximation on Linear Algebraic Groups. Transcendence Properties of the Exponential Function in Several Variables, Grundlehren Math. Wiss. 326, Springer, Berlin, 2000.

Yann Bugeaud

Université de Strasbourg

Mathématiques

7 , rue René Descartes

67084 STRASBOURG (FRANCE)

e-mail: bugeaud@math.unistra.fr 